\title{
Physiological function of myocilin and its role in the pathogenesis of glaucoma in the trabecular meshwork (Review)
}

\author{
HONGWEI WANG ${ }^{1 *}$, MINGZHE LI $^{2 *}$, ZHENZHEN ZHANG $^{3}$, HAIFENG XUE $^{4}$, XING CHEN $^{5}$ and YONG JI ${ }^{6}$ \\ ${ }^{1}$ Department of Ophthalmology, Jingjiang People's Hospital, Jingjiang, Jiangsu 214500; ${ }^{2}$ Department of Ophthalmology, \\ Shanghai Pudong Hospital, Fudan University Pudong Medical Center, Shanghai 201399; ${ }^{3}$ Department of Ophthalmology, \\ Ninth People's Hospital, Shanghai Jiao Tong University School of Medicine, Shanghai 200011; ${ }^{4}$ Public Health School, \\ Qiqihar Medical University, Qiqihar, Heilongjiang 161006; Departments of ${ }^{5}$ Science and Education \\ and ${ }^{6}$ General Surgery, Jingjiang People's Hospital, Jingjiang, Jiangsu 214500, P.R. China
}

Received June 24, 2018; Accepted November 16, 2018

DOI: $10.3892 /$ ijmm.2018.3992

\begin{abstract}
Myocilin is highly expressed in the trabecular meshwork (TM), which plays an important role in the regulation of intraocular pressure (IOP). Myocilin abnormalities may cause dysfunction of the TM, potentially leading to increased IOP. High IOP is a well-known primary risk factor for glaucoma. Myocilin mutations are common among glaucoma patients, and they are implicated in juvenile-onset open-angle glaucoma (JOAG) and adult-onset primary open-angle glaucoma (POAG). Aggregation of aberrant mutant myocilins is closely associated with glaucoma pathogenesis. The aim of the present review was to discuss the recent findings regarding the major physiological functions of myocilin, such as intra- and extracellular proteolytic processes. We also aimed to discuss the risk factors associated with myocilin and the development of glaucoma, such as misfolded/mutant myocilin, imbalance of myocilin
\end{abstract}

Correspondence to: Dr Yong Ji, Department of General Surgery, Jingjiang People's Hospital, 28 Zhongzhou Road, Jingjiang, Jiangsu 214500, P.R. China

E-mail: wangyutingwangxin@163.com

*Contributed equally

Abbreviations: JOAG, juvenile-onset open-angle glaucoma; POAG, primary open-angle glaucoma; UPR, unfolded protein response; ER, endoplasmic reticulum; TGF, transforming growth factor; CHOP, c/ebp-homologous protein; Grp78, glucose-regulated protein 78; IOP, intraocular pressure; OLF, olfactomedin; LINK, linker domain; OS, oxidative stress; IL-1, interleukin-l; ROS, reactive oxygen species; ERAD, ER-associated degradation; Grp94, glucose-regulated protein 94; NF- $\kappa \mathrm{B}$, nuclear factor $\kappa \mathrm{B}$; MMP, matrix metalloproteinase; LZ, leucine zippers; PBA, sodium 4-phenylbutyrate; MYOC, myocilin; TM, trabecular meshwork; SPARC, secreted protein acidic and rich in cysteine; TIMP3, tissue inhibitor of matrix metalloproteinase 3

Key words: myocilin, function, pathogenesis, glaucoma, trabecular meshwork and extracellular proteins, and instability of mutant myocilin associated with temperature. Finally, we further outlined certain issues that are yet to be resolved, which may represent the basis for future studies on the role of myocilin in glaucoma.

\section{Contents}

1. Introduction

2. Physiological functions and characteristics of myocilin

3. Pathogenesis of mutant $/ \mathrm{misfolded}$ myocilins

4. Imbalance of pathogenic myocilin and extracellular proteins

5. Association of glaucoma pathogenesis with the crystal structure and stability of myocilin

6. Conclusion

\section{Introduction}

Glaucoma is second leading cause of blindness after cataract, and the leading cause of irreversible blindness globally (1-4). Primary open-angle glaucoma (POAG) is the most prevalent form of glaucoma (5) and is responsible for $\sim 90 \%$ of all cases (6). The pathogenesis of glaucoma remains unknown; however, accumulating evidence indicates that genetic factors may play a causative role (7). The myocilin gene (MYOC), which encodes the secreted protein myocilin, is the first and most extensively investigated gene associated with familial forms of POAG $(8,9)$. MYOC is detected in the aqueous humor and MYOC mutations are the most common type of mutation in patients with glaucoma (10), accounting for $10 \%$ of juvenile-onset open-angle glaucoma (JOAG) (11) and 2-4\% of adult-onset POAG (12). JOAG, unlike adult-onset POAG, has a close genotype-phenotype association with regard to myocilin mutations and glaucoma, and typical signs include high intraocular pressure (IOP) $(13,14)$, severe optic nerve damage and earlier age at onset (usually <40 years) $(15,16)$, which, if left untreated, results in severe visual impairment (17).

Myocilin is ubiquitously expressed in normal tissues and organs; however, myocilin-associated disease only occurs in 
the eye (18). Myocilin is widely expressed in ocular tissues and highly expressed in the trabecular meshwork (TM) (19). Wild-type myocilin is secreted in the TM (9), is located in the aqueous humor and plays an important role in the regulation of IOP $(20,21)$. High IOP is a risk factor for glaucoma $(22,23)$ and reduction of IOP is an approach to glaucoma treatment (24). Mutant myocilin aggregation is closely associated with glaucoma (25) and it may be involved in morphological changes in the TM that may result in cell apoptosis (7). A growing body of evidence suggests that myocilin, the TM, IOP and glaucoma are closely associated.

The present review exclusively focused on the results of the latest studies that provide an insight into the physiological functions of myocilin and its role in the pathogenesis of glaucoma in the TM. An overview of the primary findings and future research topics requiring further investigation was performed in the present review.

\section{Physiological functions and characteristics of myocilin}

Structure of myocilin. Despite numerous studies over the 20 years since its discovery as a glaucoma-associated gene in 1997, the physiological functions and biological activities of myocilin in the TM remain poorly understood. The first report of identified myocilin mutations in autosomal dominant POAG came from Stone et al (26), and myocilin was found to map to the GLC1A locus at 1q24.3-q25.2 (OMIM: 601652). Myocilin, which encodes a 504-amino acid glycoprotein and undergoes glycosylation at amino acid residues 57-59 (27), has three exons and contains two major homology regions, the Nand C-terminus (Fig. 1) (23,26,28-35). Notably, the majority of myocilin mutations are localized in exon 3 (Fig. 1). The $\mathrm{N}$-terminus of myocilin contains leucine zippers (LZ) within two coil-coil domains (35). Furthermore, the N-terminus is involved in the initial myocilin oligomerization through LZ (36), and in the extracellular interactions of myocilin with other extracellular proteins through two coil-coil domains $(35,37)$. The $\mathrm{C}$-terminus contains olfactomedin (OLF), which is important for the structure and function of myocilin (35), specifically in the process of intracellular trafficking (36). Notably, N- and C-terminus functions affect the aqueous humor outflow in the TM.

Intracellular proteolytic process. Normally, myocilin is intracellularly cleaved within the endoplasmic reticulum (ER) of TM cells and secreted into the aqueous humor $(33,38)$. C-terminal myocilin fragments have been detected in the TM and the aqueous humor $(23,33)$. N-terminal myocilin containing $\mathrm{LZ}$ has also been identified (39); however, this is intracellularly retained in the ER $(23,33,36,40,41)$. N-terminal fragments can be intracellularly degraded during proteolytic processing, or they can interact with other intracellular proteins (40). Previous studies have suggested that myocilin undergoes proteolytic cleavage, and that the location of the proteolytic cleavage site is possibly between Glu214-Leu215 $(39,42)$ or between Arg226-Ile227 (23). It was reported that myocilin fragments containing OLF did not change the outflow capacity of the aqueous humor, suggesting that both OLF and LZ fragments must coexist for myocilin to function properly in the intracellular proteolytic process (39).
Similar to myocilin, calpain II (cysteine protease) is also present within the lumen of the Golgi apparatus and the ER (43). Calpain II is required for the intracellular proteolytic cleavage of myocilin (40). The proteolytic processing of myocilin does not require the N-terminus, and two different domains of myocilin participate in the proteolytic processing through calpain II (40): i) C-terminal OLF, which likely acts as a substrate binding site recognized by calpain II; and ii) linker domain (LINK), which acts as the cleavage site (Fig. 2) (40). These findings are supported by previous studies $(23,42,44)$, suggesting that myocilin mutations located at OLF may inhibit the proteolytic processing of myocilin. Amino acid positions mutated in OLF likely affect the structure of the myocilin binding site to calpain II (40). Interestingly, Pro370Leu, which contributes to the most severe glaucoma phenotype (44), produces the most severe inhibition of proteolytic processing. The inhibition of proteolytic processing by Glu323Lys and Asp380Ala is less severe, causing less severe glaucoma (23). However, the association of the severity of glaucoma with the inhibition of proteolytic processing remains unclear.

Extracellular proteolytic process. Although the physiological function of the intracellular proteolytic processing of myocilin remains unknown, the amount of proteolytic myocilin may be associated with the regulation of the normal TM structure through extracellular proteins, including fibrillin-1 (45), secreted protein acidic and rich in cysteine (SPARC) (34), hevin $(34,46)$, collagen (45), optimedin (47), decorin (45), fibronectin (48) and laminin (45,49), which contribute to the regulation of aqueous humor outflow that can affect IOP (23). The identification of proteins interacting with myocilin is a possible approach to elucidating its functions, since interacting proteins are typically involved in the same physiological and pathological processes (50).

The first report of the possible role of proteolytic processing in regulating the interactions of myocilin with itself or other extracellular proteins came from Aroca-Aguilar et al $(33,34)$, who demonstrated that homoaggregates of myocilin monomers and myocilin complexes (containing oligomers, matricellular proteins and extracellular matrix proteins) can form a dynamic extracellular network (Fig. 3) (34). The proteolytic processing of myocilin occurs in the relevant elements involved in IOP homeostasis (such as the aqueous humor and the TM); thus, the proteolytic cleavage in the dynamic myocilin network possibly participates in regulating IOP (33) through controlling IOP homeostasis (aqueous humor production and drainage). Furthermore, the myocilin network may act as a link between the extracellular matrix and matricellular proteins (34). Coincidently, OLFs in the extracellular network are similar to those resulting from intracellular proteolytic processing of myocilin through calpain II (Figs. 2 and 3) $(23,34,40)$.

Elevated IOP and overexpression of myocilin caused by inducers. Myocilin is stimulated by various inducers, including dexamethasone (DXM) (11,51-55), pentablock copolymer DXM nanoformulations (56), retinoic acid (57), transforming growth factor (TGF)- $\beta 1$ (11), TGF- $\beta 2$ (58), optineurin $(59,60)$, mechanical stretch (11), rotenone (61), and hydrogen peroxide-inducible clone-5 (62). DEX is widely 


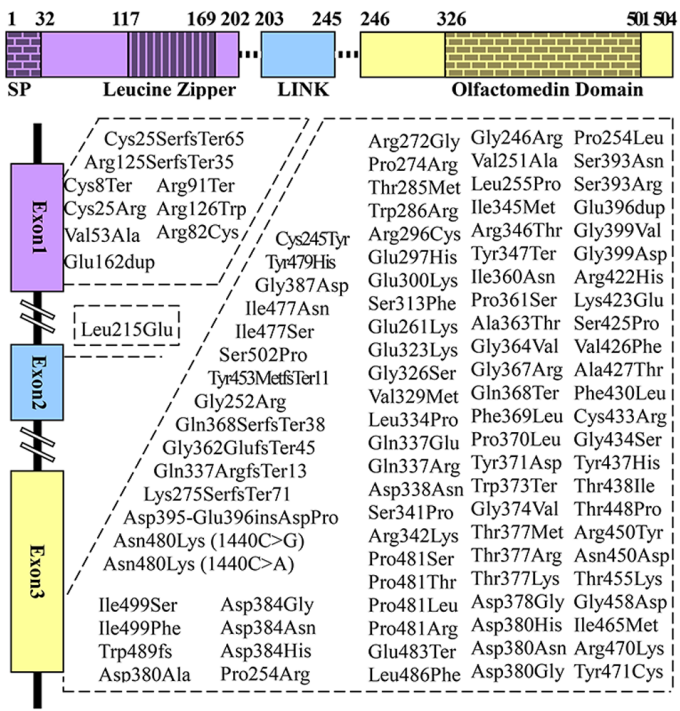

Figure 1. Structure of myocilin and pathogenic mutations localized in exons 1-3 (33). Three modules encoded by exons 1-3 approximately coincide with the N-terminus, LINK and C-terminus. SP, signal peptide; LINK, linker domain.

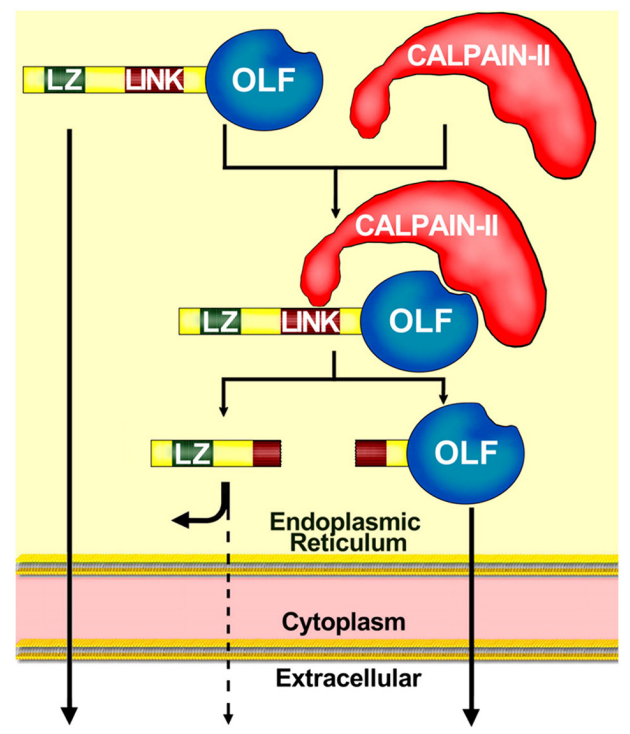

Figure 2. Proteolytic process of myocilin through calpain II (40). The proteolytic processing of myocilin is carried out by calpain II in the endoplasmic reticulum, producing two myocilin fragments: One containing LZ that is intracellularly retained and another containing OLF that is extracellularly secreted. Some full-length myocilins with LZ and OLF are also secreted. LINK contains the cleavage site. LZ, leucine zippers; OLF, olfactomedin; LINK, linker domain.

recognized as a major contributor to the induction of myocilin, which may cause high IOP.

Elevated IOP is a known primary risk factor for glaucoma $(22,23)$; however, not all cases of glaucoma exhibit high IOP $(63,64)$. Elevated IOP is caused by increased resistance to aqueous humor outflow through the TM $(65,66)$ and accounts for visual field loss; however, its pathogenesis remains unknown (67). Homeostasis of aqueous humor drainage through the TM is essential for the maintenance of normal IOP (68). DXM-induced overexpression of myocilin in the TM cells $(11,54,55)$ may increase IOP (69). Additionally, overexpression of myocilin induced by DXM possibly affects focal adhesion, stress fibers and actin reorganization in TM cells, which subsequently results in ER aggregation and TM cell stiffness, leading to increased outflow resistance (62) and elevated IOP. However, the results regarding the reversal of myocilin $(52,70)$ and increased IOP $(70,71)$ do not support the hypothesis that myocilin overexpression causes increased IOP or glaucoma; this is supported by the following data: i) Observation of an equivalent increase of IOP in $\mathrm{MYOC}^{-/-}$ and wild-type mice following DXM treatment (51); and ii) observation of increased IOP and absence of myocilin overexpression following DXM treatment (71). These studies suggest that increased IOP may not be associated with myocilin overexpression.

\section{Pathogenesis of mutant/misfolded myocilins}

The aforementioned studies suggest that the biological functions of myocilin remain poorly understood. Similarly, the role of myocilin in the pathogenesis of glaucoma is currently unknown, although its mutations have been reported to be associated with JOAG and adult-onset POAG.

Myocilin mutations. MYOC mutations alter the myocilin protein, which affects the normal regulation of IOP and may lead to glaucoma (72). To date, 278 different myocilin mutations have been reported, among which pathogenic mutations account for $37.77 \%$ (26) (Fig. 1) (23,28-35), 9 of which have been identified in exon 1,1 in exon 2 , and 95 in exon 3. Myocilin predominantly displays two types of mutations: Missense mutations $(83.8 \%)$, which are associated with JOAG and adult-onset POAG (16); and nonsense mutations (5.7\%) (26). Different myocilin mutations cause POAG with varying age at onset and glaucoma phenotypes of varying severity (73). Pro370Leu is one of the most severe glaucoma phenotypes, which is involved in mitochondrial dysfunction in TM cells and may lead to apoptosis (74). Gln368Stop is the most common mutation; however, it exhibits a markedly lower penetrance for glaucoma (75).

Autosomal dominant disorders resulting from myocilin (28) may be caused by the following three pathogenic mechanisms: The dominant negative effect, gain of function, or haploinsufficiency $(7,16)$. Some studies have demonstrated that neither haploinsufficiency $(16,19,67,76)$ nor the dominant negative effect (77) are involved in myocilin-associated pathogenicity. However, recent findings $(42,78)$ have suggested that the dominant negative effect may be involved in the pathogenic role of myocilin in glaucoma. Notably, the majority of experimental evidence support gain-of-function as a pathogenic mechanism involved in myocilin mutation-associated glaucoma $(21,23,36,37,41,42,67,73,76,79-84)$.

Pathogenesis of ER stress and oxidative stress (OS) caused by mutant myocilin. Recent studies have indicated that the notable paucity of normal myocilin $(16,67,76,85)$ or its overexpression (85) are not associated with the pathogenesis of glaucoma, and that the pathogenesis of glaucoma is dependent on the expression of mutant/misfolded myocilins $(86,87)$. Notably, mutations may cause myocilin misfolding (88). Furthermore, the secretion of wild-type myocilin is inhibited in the presence 
of co-expressed mutant myocilin $(36,41)$. The aggregation of misfolded/wild-type myocilins in the ER may be harmful for TM cells and lead to apoptosis $(19,84)$. Previous results have suggested that the TM is several times thicker in patients with glaucoma harboring mutations compared with that in patients without myocilin mutations (7). Therefore, myocilin mutations appear to be involved in the morphological changes in the TM, which lead to cell apoptosis (7).

Mutant myocilins aggregate intracellularly in insoluble and soluble aggresomes, interact with ER proteins and promote ER stress (19,81-84). Mutant myocilins have been suggested to induce apoptosis and may contribute to TM cell dysfunction, leading to increased IOP $(86,89,90)$. Subsequently, ER stress may cause OS (87). During ER stress, the level of reactive oxygen species (ROS) is increased (91). Excess production and an accumulation of ROS compromise reduction-oxidation balance and cause OS (91). Notably, ER stress and OS are associated events involved in the pathogenesis of various diseases (91), including glaucoma-associated TM damage and increased IOP $(87,90)$. However, the molecular pathways that connect ER stress and OS are poorly understood. OS is caused by the excessive production and aggregation of ROS (91). The $\mathrm{TM}$, which is the most sensitive to OS among the tissues in the anterior chamber of the eye (86), comes into direct contact with the ROS-containing aqueous humor (87). Furthermore, ROS alone may cause protein misfolding (86). Misfolded myocilin can render cells more sensitive to OS (87). It was recently suggested that mutant myocilin inhibited antioxidant enzymes, such as paraoxonase 2 , that efficiently decrease OS and inhibit ER stress-induced apoptosis (87). Therefore, decreased antioxidative enzymes in the TM may cause ROS elevation in the aqueous humor outflow system (87).

It has been reported that OS aggravates ER stress by inducing the overproduction of misfolded proteins (92). OS has also been considered a major factor causing damage to the TM (93). To relieve the damage from ER stress and restore homeostasis, the aggregated misfolded myocilin activates the unfolded protein response (UPR), which protects the TM cells (94-96), improves the protein folding mechanism and degrades misfolded proteins $(94,95)$. However, if ER stress persists, UPR induces cell apoptosis $(19,84)$. In addition, mutant myocilin cannot be cleared by ER-associated degradation, which transports degraded products of misfolded myocilin to the cytosol (19), leading to deleterious aggregation of amyloid-containing myocilin (97). These studies suggest that myocilin misfolding, UPR, ROS, OS and ER stress may be related events. Notably, ER stress and OS are risk factors for glaucoma, and together with the deleterious effect of misfolded myocilin, may cause a more severe glaucoma phenotype compared with any of these factors alone. These associated events disrupt the proteostasis of myocilin, leading to an imbalance between production and clearance of misfolded myocilins.

Pathogenesis of the co-aggregation of glucose-regulated protein (Grp) 94 with mutant myocilin. It has been reported that the inhibition of Grp94 is an effective approach to the treatment of glaucoma $(79,98)$, supporting the co-aggregation of Grp94 with mutant myocilin and leading to retention within the ER (Fig. 4) (80). Not only does Grp94 accelerate the myocilin-OLF

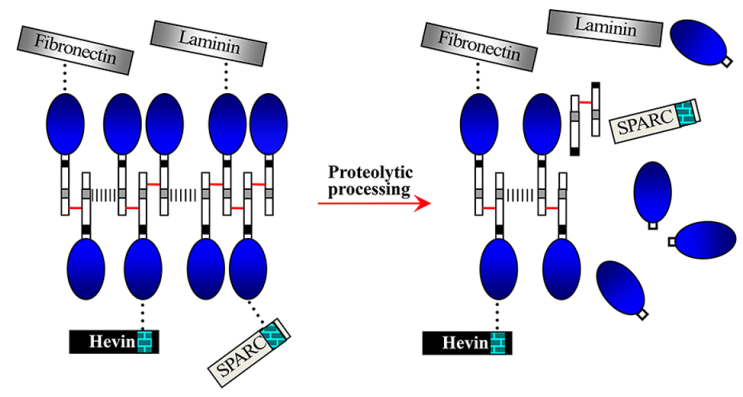

Figure 3. Interactions of myocilin with extracellular matrix proteins (laminin and fibronectin) and matricellular proteins (SPARC and hevin) (34). Homoaggregates of myocilin monomers covalently interact through disulfide bonds (short red lines) within LZ (12). Myocilin complexes interact by noncovalent bonds (grey dashed lines) in N-terminus (rectangle linked to the blue oval). Full-length myocilins non-covalently (black dots) interact with the extracellular calcium binding domains (brick pattern) of SPARC and hevin through OLF (blue oval). Interacting fashion of myocilin with laminin and fibronectin could be similar to that of SPARC and hevin. SPARC, secreted protein acidic and rich in cysteine; LZ, leucine zippers; OLF, olfactomedin.

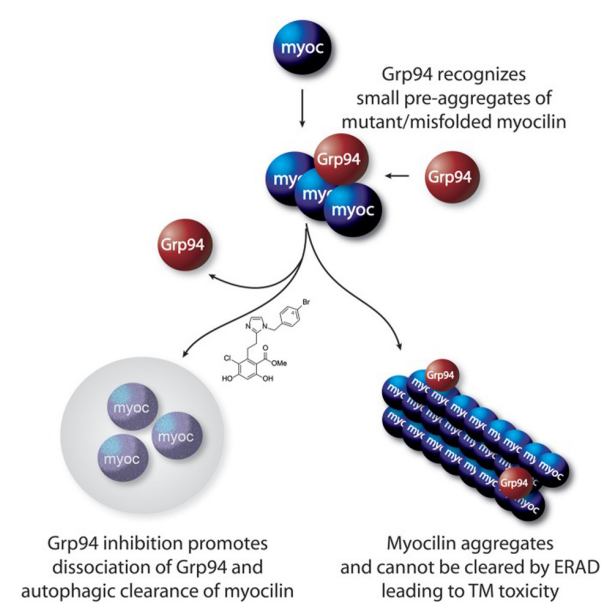

Figure 4. Co-aggregation of Grp94 with mutant/misfolded myocilin (80). Grp94, glucose-regulated protein 94 .

aggregation rate, but it also enhances co-precipitation with OLF $(79,80,99)$. Therefore, Grp94 inhibition facilitates mutant myocilin clearance as an anti-glaucoma therapy $(79,97,100)$. 4-Br-BnIm, a Grp94 inhibitor, significantly clears aggregated myocilin caused by overexpression mutations (97), and alleviates mutant myocilin-induced toxicity against TM cells $(27,79)$ via a secondary autophagic pathway to facilitate clearance (27). When forced to misfold and aggregate, wild-type myocilin becomes a client of Grp94 and sensitized to 4-Br-BnIm (79). Recent studies $(12,101)$ reported that myocilin aggregates were cleared by a ubiquitin-proteasome and autophagy lysosomal pathways under normal homeostatic conditions. However, when myocilin is mutated, autophagy is activated due to dysfunction of the proteasomal degradation pathway (12), and mutant myocilin is preferably degraded by autophagy (101). Grp94 inhibitors prevent Grp94 from aggregating with mutant myocilin, which induces a secondary autophagic pathway to promote clearance of abnormal myocilin (80). Grp94 is also a regulator of UPR (79). Therefore, a reduction in Grp94 may affect UPR, which induces TM cell death under persistent ER stress due to abnormal aggregations of Grp94 and misfolded myocilin. 
A

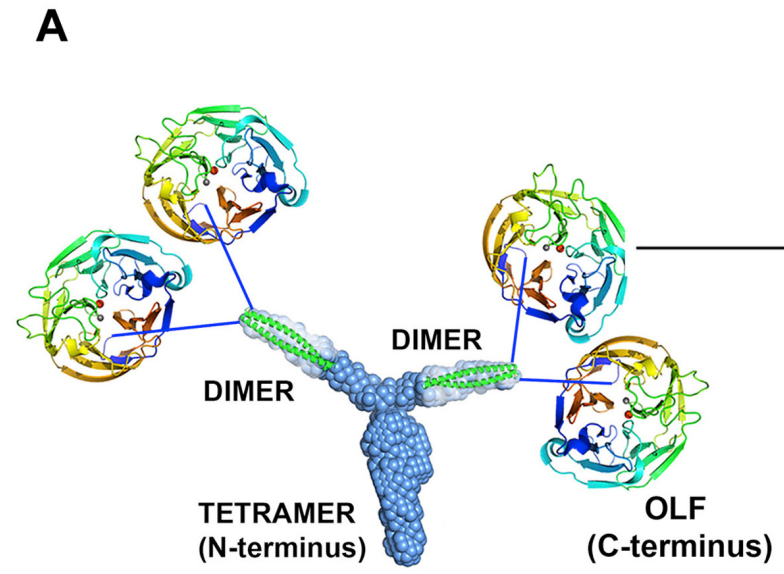

B

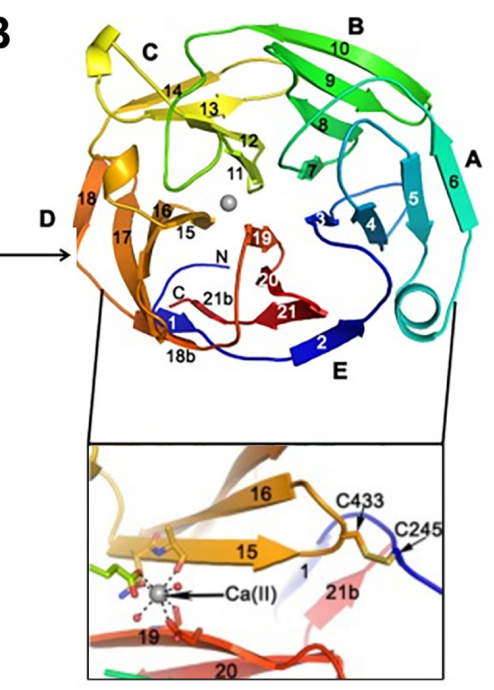

Figure 5. Structure of myocilin $(108,117)$. (A) The N-terminus has a Y-shaped parallel (117). (B) The crystal of myocilin-OLF is a five-bladed $\beta$-propeller, and each blade is composed of four antiparallel $\beta$-strands, arranged radically around a central water-filled cavity (108). The blades in OLF are notably asymmetric. Nearly half of the toroid-shaped molecule is occupied by blades D and E. The myocilin-OLF propeller has five blades (A, B, C, D and E). A disulfide bond and calcium-binding site are located at the bottom face of the propeller, and a single disulfide bond is observed between Cys433 located within Loop D-15/D-16 and Cys245 at the N-terminus prior to the start of E-1. Tyr 437 further stabilizes the region near the single disulfide bond. Unlike other amyloid-like proteins (120), the effect of disulfide bonds on the stability of wild-type myocilin is not significant.

Myocilin and the interleukin (IL)-1/nuclear factor (NF)- $\kappa B$ inflammatory stress signaling pathway. Activation of IL-1/NF- $\kappa B$ inflammatory stress is a defining characteristic of high-tension glaucoma (102). The release of potent proinflammatory IL-1, including IL-1 $\beta$, may lead to inflammation (103) through activation of the NF- $\mathrm{KB}$ pathway, which induces an inflammatory response (104). It has been demonstrated that chronic low-grade inflammation causes ocular hypertension (105) and is also involved in the pathogenesis of glaucoma (103). Mutant myocilins aggregate within the TM cells and activate the NF- $\mathrm{KB}$ signaling pathway, significantly inducing the expression of IL-1 and IL-1 $\beta$; however, extracellular mutant myocilin does not activate the NF- $\mathrm{kB}$ signaling pathway (106). Therefore, intracellular aggregations of mutant myocilins may induce the overexpression of IL-1 via activating NF- $\mathrm{kB}$, leading to chronic inflammation that can cause elevated IOP.

Interestingly, IL-1 has additional beneficial effects on glaucoma, including reducing IOP through stimulation of matrix metalloproteinase (MMP) expression (106) and inhibiting apoptosis caused by OS through NF- $\mathrm{KB}$ (102). IOP and $O S$ are associated with the aggregation of misfolded/mutant myocilins (86). Notably, IL-1 was increased by 10 -fold in TM cells harboring Gln368Stop; however, the increase was only 6 -fold in cells harboring Try437His (106). These findings suggest that abnormal aggregation of myocilin mutations may induce OS and upregulate IL-1. However, the amount of IL-1 induced by Gln368Stop is notably higher compared with that induced by Try437His. Coincidently, Try437His causes a severe glaucoma phenotype; however, Gln368Stop only causes a moderately severe glaucoma phenotype. This may be explained by the greater extent of IOP reduction and inhibition of the apoptotic process caused by OS through IL-1 via Gln368Stop compared with Try437His in the short-term. This also explained the finding that Gln368Stop is the most common mutation; however, it revealed a markedly lower penetrance for glaucoma caused by high IOP.

\section{Imbalance of pathogenic myocilin and extracellular proteins}

Glaucoma-associated TM damage is involved in various biochemical and morphological changes, including aberrant extracellular matrix protein aggregation and cell apoptosis (107). Synthesis and degradation of the extracellular matrix are in a dynamic balance that is constantly remodeled by proteolysis and protein deposition (52). Certain diseases, including glaucoma, may result from disruption of this dynamic balance (107). Recently, the crystal structure of myocilin-OLF was elucidated, and it indicated that myocilin-OLF belongs to the five-bladed $\beta$-propeller family (108), which is a known hub for protein-protein interactions. To date, a number of extracellular proteins interacting with myocilin have been identified, including tissue inhibitor of matrix metalloproteinase (TIMP3) $(8,50)$, fibronectin (107), flotillin-1 (109) and hevin (46).

Effect of pathogenic myocilin on MMPs and MMP inhibitors. MMPs have multiple physiological functions, among which cell migration and proliferation have been linked to myocilin $(110,111)$. Changes in MMP activity are involved in the pathogenesis of glaucoma (112). Notably, MMP2 activity may be associated with the regulation of IOP, which may be predominantly associated with the TM, where myocilin and TIMP3 coexist (50). MMP2, which is abundant in the TM (8), is involved in the breakdown of extracellular matrix (52), which facilitates aqueous humor outflow (8). It has also been demonstrated that myocilin mutations or MYOC null can enhance inhibition of MMP2 by TIMP3 $(8,50)$. An imbalance between MMPs and TIMP within the TM can 


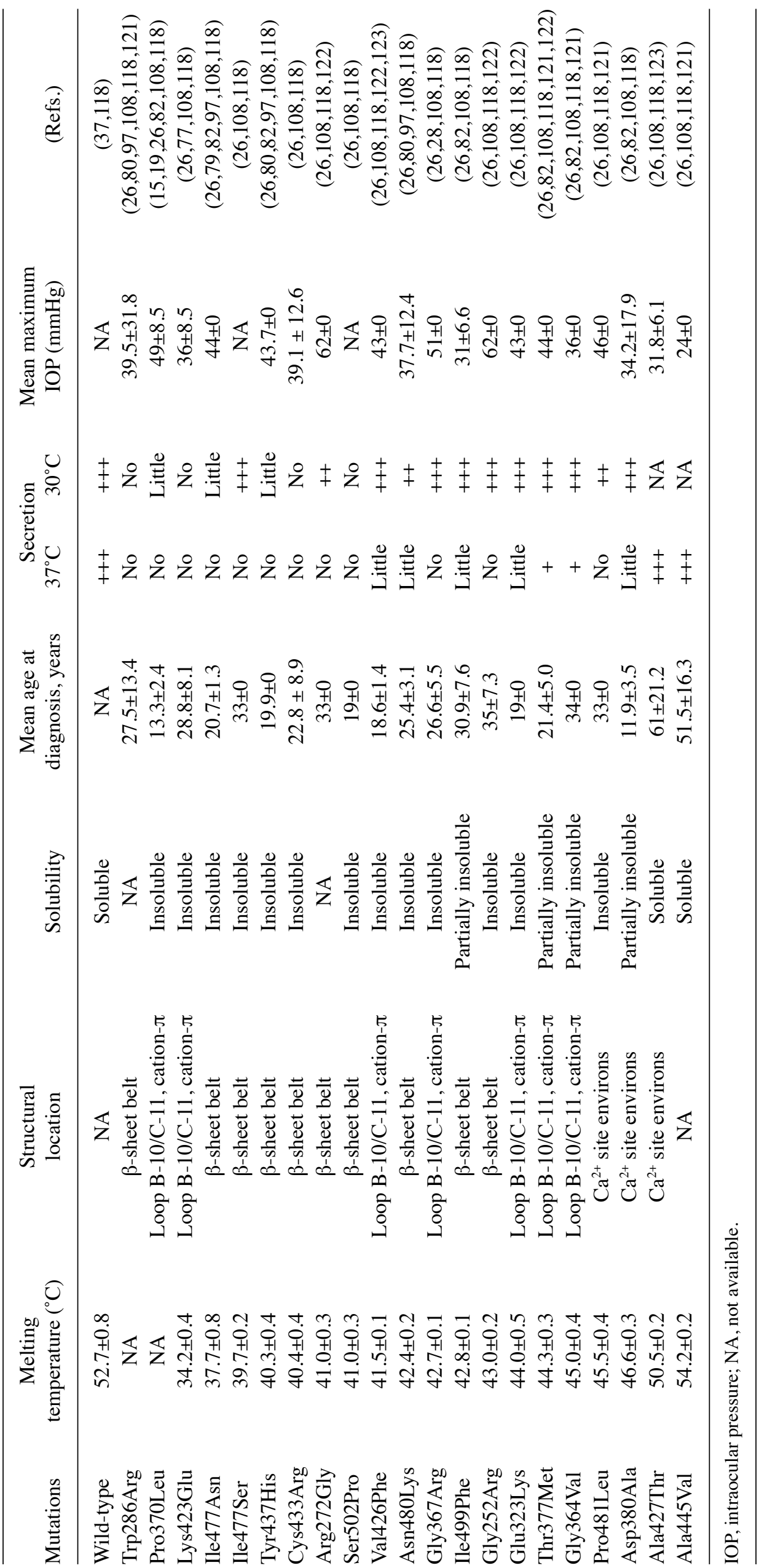


cause POAG (113). Of note, mutant myocilins can also reduce the active forms of MMP2 (107). Therefore, the effect of mutant myocilin on MMP2 activation disrupts the balance between MMPs and TIMPs, leading to glaucoma (96). In addition, reduction of MMP2 inhibits the decomposition of the extracellular matrix, disrupting its homeostasis, which may change the TM morphology and function, leading to increased IOP.

Effect of pathogenic myocilin on fibronectin, flotillin-1 and hevin. Fibronectin is a key component of the extracellular matrix of the TM, and is also an important mediator involved in extracellular matrix formation, which regulates aqueous humor outflow (114). Aberrant aggregation of the extracellular matrix in the TM reduces aqueous humor outflow and increases IOP in POAG (107). Overexpression of fibronectin and increased ER stress markers c/ebp-homologous protein (CHOP) and Grp78 coexist in mice harboring MYOC-Tyr437His (107). Furthermore, another study reported that mutant myocilins increased CHOP and Grp78 in the TM (96). Myocilin, fibronectin, CHOP and Grp78 expression levels were increased following DXM treatment (115), which was similar to the overexpression of myocilin and fibronectin observed following DXM-Ac treatment (51). Recent studies have suggested that a reduction of ER stress through sodium 4-phenylbutyrate decreases the DXM-induced increase of IOP (115) and fibronectin aggregation caused by mutant myocilin (107) in TM cells. Therefore, myocilin, fibronectin, CHOP, Grp78 and the extracellular matrix may be considered as elements implicated in the pathogenesis of glaucoma in the TM. Additionally, mutant myocilin induces aberrant aggregation of the extracellular matrix in the ER of TM cells, which may contribute to decreased aqueous humor outflow and increased IOP (107). Furthermore, failure of the TM to reduce ER stress caused by mutant myocilin accounts for the induction of CHOP, which may also be associated with apoptosis and increased IOP in the TM cells (96).

Flotillin-1, a structural protein of lipid rafts, interacts with myocilin (109). Myocilin mutations, including Gly364Val, Tyr437His and Lys423Glu, which are scattered in the OLF, fail to interact with flotillin-1 (109), suggesting that myocilin-OLF may be required for the interaction. Although the role of the interaction remains to be elucidated, loss of interaction with flotillin-1 due to myocilin mutations may be a pathogenic mechanism underlying the development of POAG (109). Hevin is a matricellular protein involved in the assembly of the extracellular matrix (116). Mutant myocilin causes intracellular aggregation of hevin and also affects hevin secretion (46). Hevin coexpressed with Pro370Leu, which is also known as a severe glaucoma mutation, aggravates the impairment of hevin secretion (46). These findings indicate that mutant myocilins affect the interactions with extracellular proteins, leading to disruption of extracellular matrix homeostasis, which may be involved in the pathogenesis of glaucoma.

\section{Association of glaucoma pathogenesis with the crystal structure and stability of myocilin}

Crystal structure of myocilin. A better understanding of the conformation of myocilin can provide a structural basis for investigating myocilin mis-/unfolding in myocilin-associated glaucoma and, in turn, result in a better understanding of myocilin-associated glaucoma pathogenesis. The myocilin $\mathrm{N}$-terminus has a unique tripartite architecture, including a Y-shaped parallel dimer-of-dimers with distinct tetramer and dimer regions. Furthermore, full-length myocilin should also be branched, with two pairs of C-terminal OLFs (Fig. 5A) (117). MYOC-Glu396Asp has discontinuous D-18/D-18b and E-21/ E-21b strands (Fig. 5B) $(108,117)$ that are unlike the continuous D-18 and E-21 strands in wild-type myocilin. Accumulating evidence has revealed that pathogenic myocilin mutations are associated with intracellular aggregation propensity and thermal stability $(19,32,37,41,42,82,108,118,119)$. Three destabilizing regions are localized in myocilin-OLF (108): i) Loop B-10/C-11 and cation- $\pi$; ii) hydrophobic $\beta$-sheet belt; and iii) $\mathrm{Ca}^{2+}$ site environs (Fig. 5B).

Structural location and stability of myocilin mutations. The largest number of mutations are observed in core $\beta$-sheet belts $(\sim 40 \%)$, loop B-10/C-11 and cation- $\pi(\sim 33 \%)$ (108), particularly the most destabilized myocilin-OLF mutations, such as Trp286Arg, Tyr437His, Lys423Glu, Pro370Leu, Ile477Asn and Ile477Ser $(118,119)$. The myocilin-OLF mutations exhibit changes in the side chains. The most destabilizing mutations also exhibit other characteristics: i) Lower melting temperature (Table I) (108); ii) insoluble aggregates (118); and iii) earlier age at diagnosis. In contrast to the most destabilizing mutations, the most stabilizing myocilin-OLF mutations, Ala427Thr and Ala445Val (118), exhibit similar stability and structure to those of wild-type myocilin-OLF. Furthermore, the difference in their melting temperature from that of wild-type myocilin-OLF $\left(52.7 \pm 0.8^{\circ} \mathrm{C}\right)$ is only within $2^{\circ} \mathrm{C}$, and they are soluble (118). The trend in melting temperature follows the general stability of myocilin-OLF mutations and the severity of their aggregation. Additionally, this instability may cause structural changes in myocilin-OLF, resulting in mis-/unfolding. Notably, higher proportion of mis-/unfolded myocilin has been associated with greater exposure of interior hydrophobic regions and more severe intracellular aggregation propensity (118). Intracellular aggregation of mutant myocilin deforms TM cells (84) and changes the size of pores between the cells, through which aqueous humor outflows. Dysfunction of TM cells may contribute to the pathogenesis of glaucoma $(41,84)$.

Myocilin mutations at a moderate level of stability (Table I) renew the secretion through shifting temperature from 37 to $30^{\circ} \mathrm{C}(37,82,118)$, demonstrating their temperature-sensitive secretion. Trimethylamine N-oxide and sarcosine also stabilize myocilin mutations through shifting their melting temperatures to near that of wild-type myocilin (119). Thr377Met, Ile499Phe and Gly364Val, which are abundantly secreted at $30^{\circ} \mathrm{C}$, are associated with less severe glaucoma (82). Interestingly, Pro370Leu and Ile477Asn, which are associated with a more severe glaucoma phenotype $(82,118)$, are not secreted at higher levels when the temperature changes (82). Thus, temperature-sensitive secretion may be a notable property of these moderate myocilin mutations (82). A temperature of $30^{\circ} \mathrm{C}$ is a condition known to facilitate protein folding $(37,42)$. Therefore, this favorable temperature promotes the correct folding of myocilin into its native form. Taken together, these 
studies demonstrated that the stability of myocilin mutations may be associated with their pathogenicity. Notably, the less stable myocilin mutations were associated with the more severe glaucoma phenotype and were less sensitive to temperature.

\section{Conclusion}

Based on the aforementioned evidence, although myocilin mutations have been reported to be associated with JOAG and adult-onset POAG, the physiological functions and pathogenicity of myocilin remain elusive. Thus far, there are several possible points of view on the pathogenic potential of myocilin: i) Myocilin misfolding/unfolding; (ii) overexpression of myocilin; iii) co-aggregation of Grp94; iv) disruption of extracellular matrix homeostasis caused by mutant myocilin; v) OS, ER stress and IL-1/NF- $\mathrm{KB}$ inflammatory stress; and vi) instability resulting from conformational disorders caused by mutant myocilin. However, certain suggestions require further investigation, particularly those with contradicting conclusions. Furthermore, unsolved or partially solved problems require further research, as they could direct future studies on myocilin and contribute to novel therapeutic approaches to the treatment of myocilin-associated glaucoma.

The functions of myocilin through interactions of its binding partners should be further investigated, as interacting proteins may have similar biological functions. In addition, it is necessary to gain further insight into the proteolytic processes of myocilin, as this may contribute to an effective approach to breaking down abnormal aggregations of myocilin. Furthermore, a more detailed understanding of the structural basis of myocilin stability will be valuable for elucidating the roles of conformational disorders, such as misfolding or unfolding, in myocilin-associated glaucoma. Notably, this may help elucidate the role of myocilin mutations in the pathogenesis of glaucoma. Additionally, the association of glaucoma phenotype severity with the secretion (associated with temperature, stability and insolubility) of mutant myocilin should be emphasized, as unraveling these elusive associations may contribute to understanding the pathogenesis underlying abnormal aggregations of mutant myocilin. Future studies regarding myocilin should focus on the deleterious effect of myocilin misfolding on OS and ER stress in the TM, which are a series of associated events, each of which causes the next, leading to further injury to the TM. Furthermore, these effects are associated with increased IOP, which is a known primary risk factor for glaucoma.

\section{Acknowledgements}

The authors would like to thank Xin Wang in the Library of Qiqihar Medical University for the careful edits and the knowledge promoted and supported by the Heilongjiang Province Philosophy and Social Science Research Planning project (16TQB04).

\section{Funding}

The present study was supported by a grant from Taizhou Science and Technology Support Projects for Social Development (2016) of Taizhou Science and Technology Bureau (SSF20160112).

\section{Availability of data and materials}

Not applicable.

\section{Authors' contributions}

HW, ML, ZZ, HX, XC and YJ designed the article, contributed to the conception of the study and critically revised the article for important intellectual content. HW and YJ drafted the article. All authors read and approved the final manuscript.

\section{Ethics approval and consent to participate}

Not applicable.

\section{Patient consent for publication}

Not applicable.

\section{Competing interests}

The authors declare that they have no competing interests.

\section{References}

1. Khawaja AP, Cooke Bailey JN, Wareham NJ, Scott RA, Simcoe M, Igo RP Jr, Song YE, Wojciechowski R, Cheng CY, Khaw PT, et al: Genome-wide analyses identify 68 new loci associated with intraocular pressure and improve risk prediction for primary open-angle glaucoma. Nat Genet 50: 778-782, 2018.

2. Wang X, Huai G, Wang H, Liu Y, Qi P, Shi W, Peng J, Yang H, Deng $S$ and Wang Y: Mutual regulation of the Hippo/Wnt/LPA/TGF- $\beta$ signaling pathways and their roles in glaucoma (Review). Int J Mol Med 41: 1201-1212, 2018.

3. Rangachari K, Bankoti N, Shyamala N, Michael D, Sameer Ahmed Z, Chandrasekaran P and Sekar K: Glaucoma Pred: Glaucoma prediction based on myocilin genotype and phenotype information. Genomics S0888-S7543: 30087-30089, 2018.

4. Narooie-Nejad M, Rasouli A, Mousavi M and Rohani MR: Study of MYOC gene mutation in POAG patients in zahedan iran. Clin Lab 63: 1283-1291, 2017.

5. Rasnitsyn A, Doucette L, Seifi M, Footz T, Raymond V and Walter MA: FOXC1 modulates MYOC secretion through regulation of the exocytic proteins RAB3GAP1, RAB3GAP2 and SNAP25. PLoS One 12: e0178518, 2017.

6. Sharma S, Bollinger KE, Kodeboyina SK, Zhi W, Patton J, Bai S, Edwards B, Ulrich L, Bogorad D and Sharma A: Proteomic alterations in aqueous humor from patients with primary open angle glaucoma. Invest Ophthalmol Vis Sci 59: 2635-2643, 2018.

7. Hamanaka T, Kimura M, Sakurai T, Ishida N, Yasuda J, Nagasaki M, Nariai N, Endo A, Homma K, Katsuoka F, et al: A histologic categorization of aqueous outflow routes in familial open-angle glaucoma and associations with mutations in the MYOC gene in japanese patients. Invest Ophthalmol Vis Sci 58: 2818-2831, 2017.

8. Fini ME: Another piece of the puzzle: MYOC and myocilin glaucoma. Invest Ophthalmol Vis Sci 58: 5319, 2017.

9. Donegan RK and Lieberman RL: Discovery of molecular therapeutics for glaucoma: Challenges successes and promising directions. J Med Chem 59: 788-809, 2016.

10. Katoli P, Godbole A, Romanowski MJ, Clark K, Meredith E, Saenz-Vash V, Wang YK, Lewicki N, Nguyen AA and Lynch JM: Full-length myocilin protein is purified from mammalian cells as a dimer. Protein Expr Purif 147: 38-48, 2018.

11. Faralli JA, Clark RW, Filla MS and Peters DM: NFATc1 activity regulates the expression of myocilin induced by dexamethasone. Exp Eye Res 130: 9-16, 2015.

12. Qiu Y, Shen X, Shyam R, Yue BY and Ying H: Cellular processing of myocilin. PLoS One 9: 92845, 2014.

13. Gupta V, Somarajan BI, Gupta S, Chaurasia AK, Kumar S, Dutta P, Gupta V, Sharma A, Tayo BO and Nischal K: The inheritance of juvenile onset primary open angle glaucoma. Clin Genet 92: 134-142, 2017. 
14. Mauri L, Uebe S, Sticht H, Vossmerbaeumer U, Weisschuh N, Manfredini E, Maselli E, Patrosso M, Weinreb RN, Penco S, et al: Expanding the clinical spectrum of COL1A1 mutations in different forms of glaucoma. Orphanet J Rare Dis 11: 108, 2016.

15. Huang C, Xie L, Wu Z, Cao Y, Zheng Y, Pang CP and Zhang M: Detection of mutations in MYOC OPTN NTF4 WDR36 and CYP1B1 in Chinese juvenile onset open-angle glaucoma using exome sequencing. Sci Rep 8: 4498-4505, 2018.

16. Wiggs JL and Vollrath D: Molecular and clinical evaluation of a patient hemizygous for TIGR/MYOC. Arch Ophthalmol 119: $1674-1678,2001$.

17. Gupta V, Ganesan VL, Kumar S, Chaurasia AK, Malhotra S and Gupta S: Visual disability among juvenile open-angle glaucoma patients. J Glaucoma 27: e87-e89, 2018.

18. Borrás T: The effects of myocilin expression on functionally relevant trabecular meshwork genes: A mini-review. J Ocul Pharmacol Ther 30: 202-212, 2014.

19. Liu Y and Vollrath D: Reversal of mutant myocilin non-secretion and cell killing: Implications for glaucoma. Hum Mol Genet 13 : 1193-1204, 2004.

20. Hernandez H, Millar JC, Curry SM, Clark AF and McDowell CM: BMP and activin membrane bound inhibitor regulates the extracellular matrix in the trabecular meshwork. Invest Ophthalmol Vis Sci 59: 2154-2166, 2018.

21. Jain A, Zode G, Kasetti RB, Ran FA, Yan W, Sharma TP, Bugge K, Searby CC, Fingert JH, Zhang F, et al: CRISPR-Cas9-based treatment of myocilin-associated glaucoma. Proc Natl Acad Sci USA 114: 11199-11204, 2017.

22. Kim JH and Caprioli J: Intraocular pressure fluctuation: Is it important? J Ophthalmic Vis Res 13: 170-174, 2018.

23. Aroca-Aguilar JD, Sánchez-Sánchez F, Ghosh S, Coca-Prados M and Escribano J: Myocilin mutations causing glaucoma inhibit the intracellular endoproteolytic cleavage of myocilin between amino acids Arg226 and Ile227. J Biol Chem 280: 21043-21051, 2005.

24. Jiang L, Eaves S, Dhillon N and Ranjit P: Postoperative outcomes following trabeculectomy and nonpenetrating surgical procedures: A 5-year longitudinal study. Clin Ophthalmol 12: 995-1002, 2018

25. Wang Y, Gao Y, Hill SE, Huard DJE, Tomlin MO, Lieberman RL, Paravastu AK and Hall CK: Simulations and experiments delineate amyloid fibrilization by peptides derived from glaucoma-associated myocilin. J Phys Chem B 122 5845-5850, 2018.

26. Hewitt AW, Mackey DA and Craig JE: Myocilin myocilin allele-specific glaucoma phenotype database. Hum Mutat 29: 207-211, 2008

27. Stothert AR, Fontaine SN, Sabbagh JJ and Dickey CA: Targeting the ER-autophagy system in the trabecular meshwork to treat glaucoma. Exp Eye Res 144: 38-45, 2016.

28. Yao YH, Wang YQ, Fang WF, Zhang L, Yang JH and Zhu YH: A recurrent G367R mutation in MYOC associated with juvenile open angle glaucoma in a large chinese family. Int J Ophthalmol 11: 369-374, 2018.

29. Souzeau E, Burdon KP, Ridge B, Dubowsky A, Ruddle JB and Craig JE: A novel de novo myocilin variant in a patient with sporadic juvenile open angle glaucoma. BMC Med Genet 17: 30 , 2016.

30. Wang F, Li Y, Lan L, Li B, Lin L, Lu X and Li J: Ser341Pro MYOC gene mutation in a family with primary open-angle glaucoma. Int J Mol Med 35: 1230-1236, 2015.

31. Yang Y, Shi Y, Huang X, Li X, Ye Z, Shuai P, Qu C, Chen R, $\mathrm{Xu}$ J, Yang Z, et al: Identification of a novel MYOC mutation in a Chinese family with primary open-angle glaucoma. Gene 571: 188-193, 2015

32. Zadoo S, Nguyen A, Zode G and Hulleman JD: A novel luciferase assay for sensitively monitoring myocilin variants in cell culture. Invest Ophthalmol Vis Sci 57: 1939-1950, 2016.

33. Aroca-Aguilar JD, Martínez-Redondo F, Sánchez-Sánchez F, Coca-Prados M and Escribano J: Functional role of proteolytic processing of recombinant myocilin in self-aggregation. Invest Ophthalmol Vis Sci 51: 72-78, 2010

34. Aroca-Aguilar JD, Sánchez-Sánchez F, Ghosh S Fernández-Navarro A, Coca-Prados $\mathrm{M}$ and Escribano $\mathrm{J}$ : Interaction of recombinant myocilin with the matricellular protein SPARC: Functional implications. Invest Ophthalmol Vis Sci 52: 179-189, 2011

35. Resch ZT and Fautsch MP: Glaucoma-associated myocilin: A better understanding but much more to learn. Exp Eye Res 88: 704-712, 2009
36. Caballero M, Rowlette LL and Borras T: Altered secretion of a TIGR/MYOC mutant lacking the olfactomedin domain. Biochim Biophys Acta 1502: 447-460, 2000.

37. Gobeil S, Letartre L and Raymond V: Functional analysis of the glaucoma-causing TIGR/myocilin protein: Integrity of amino-terminal coiled-coil regions and olfactomedin homology domain is essential for extracellular adhesion and secretion. Exp Eye Res 82: 1017-1029, 2006.

38. Zhou T, Souzeau E, Sharma S, Landers J, Mills R, Goldberg I, Healey PR, Graham S, Hewitt AW, Mackey DA, et al: Whole exome sequencing implicates eye development the unfolded protein response and plasma membrane homeostasis in primary open-angle glaucoma. PLoS One 12: e0172427, 2017.

39. Goldwich A, Ethier CR, Chan DW and Tamm ER: Perfusion with the olfactomedin domain of myocilin does not affect outflow facility. Invest Ophthalmol Vis Sci 44: 1953-1961, 2003.

40. Sánchez-Sánchez F, Martínez-Redondo F, Aroca-Aguilar JD, Coca-Prados $\mathrm{M}$ and Escribano J: Characterization of the intracellular proteolytic cleavage of myocilin and identification of calpain II as a myocilin-processing protease. J Biol Chem 282: 27810-27824, 2007.

41. Jacobson N, Andrews M, Shepard AR, Nishimura D, Searby C, Fingert JH, Hageman G, Mullins R, Davidson BL, Kwon YH, et al: Non-secretion of mutant proteins of the glaucoma gene myocilin in cultured trabecular meshwork cells and in aqueous humor Hum Mol Genet 10: 117-125, 2001.

42. Aroca-Aguilar JD, Sánchez-Sánchez F, Martínez-Redondo F, Coca-Prados $\mathrm{M}$ and Escribano J: Heterozygous expression of myocilin glaucoma mutants increases secretion of the mutant forms and reduces extracellular processed myocilin. Mol Vis 14: 2097-2108, 2008.

43. Hood JL, Brooks WH and Roszman TL: Differential compartmentalization of the calpain/calpastatin network with the endoplasmic reticulum and Golgi apparatus. J Biol Chem 279: 43126-43135, 2004.

44. Wei YT, Li YQ, Bai YJ, Wang M, Chen JH, Ge J and Zhuo YH: Pro370Leu myocilin mutation in a chinese pedigree with juvenile-onset open angle glaucoma. Mol Vis 17: 1449-1456, 2011

45. Ueda J, Wentz-Hunter K and Yue BY: Distribution of myocilin and extracellular matrix components in the juxtacanalicular tissue of human eyes. Invest Ophthalmol Vis Sci 43: 1068-1076, 2002.

46. Li Y, Aroca-Aguilar JD, Ghosh S, Sánchez-Sánchez F, Escribano $J$ and Coca-Prados M: Interaction of myocilin with the C-terminal region of hevin. Biochem Biophys Res Commun 339: 797-804, 2006.

47. Torrado M, Trivedi R, Zinovieva R, Karavanova I and Tomarev SI: Optimedin: A novel olfactomedin-related protein that interacts with myocilin. Hum Mol Genet 11: 1291-1301, 2002

48. Filla MS, Liu X, Nguyen TD, Polansky JR, Brandt CR, Kaufman PL and Peters DM: In vitro localization of TIGR/MYOC in trabecular meshwork extracellular matrix and binding to fibronectin. Invest Ophthalmol Vis Sci 43: 151-161, 2002.

49. Fautsch MP, Vrabel AM and Johnson DH: The identification of myocilin-associated proteins in the human trabecular meshwork. Exp Eye Res 82: 1046-1052, 2006.

50. Joe MK, Lieberman RL, Nakaya N and Tomarev SI: Myocilin regulates metalloprotease 2 activity through interaction with TIMP3. Invest Ophthalmol Vis Sci 58: 5308-5318, 2017.

51. Patel GC, Phan TN, Maddineni P, Kasetti RB, Millar JC, Clark AF and Zode GS: Dexamethasone-induced ocular hypertension in mice: Effects of myocilin and route of administration. Am J Pathol 187: 713-723, 2017.

52. Li G, Cui G, Dismuke WM, Navarro I,Perkumas K, Woodward DF and Stamer WD: Differential response and withdrawal profile of glucocorticoid-treated human trabecular meshwork cells. Exp Eye Res 155: 38-46, 2017.

53. Webber HC, Bermudez JY, Sethi A, Clark AF and Mao W: Crosstalk between TGF $\beta$ and Wnt signaling pathways in the human trabecular meshwork. Exp Eye Res 148: 97-102, 2016.

54. Raghunathan VK, Morgan JT, Park SA, Weber D, Phinney BS, Murphy CJ and Russell P: Dexamethasone stiffens trabecular meshwork trabecular meshwork cells and matrix. Invest Ophthalmol Vis Sci 56: 4447-4459, 2015.

55. Nguyen TD, Chen P, Huang WD, Chen H, Johnson D and Polansky JR: Gene structure and properties of myocilin an olfactomedin-related glycoprotein cloned from glucocorticoid-induced trabecular meshwork cells. J Biol Chem 273: 6341-6350, 1998. 
56. Agrahari V,Li G, Agrahari V, Navarro I, Perkumas K, Mandal A, Stamer WD and Mitra AK: Pentablock copolymer dexamethasone nanoformulations elevate MYOC: In vitro liberation, activity and safety in human trabecular meshwork cells. Nanomedicine (Lond) 12: 1911-1926, 2017.

57. Prat C, Belville C, Comptour A, Marceau G, Clairefond G, Chiambaretta F, Sapin V and Blanchon L: Myocilin expression is regulated by retinoic acid in the trabecular meshwork-derived cellular environment. Exp Eye Res 155: 91-98, 2017.

58. Wu Y, Chen W, Guo M, He Q and Hu Y: Effects of transforming growth factor- $\beta 2$ on myocilin expression and secretion in human primary cultured trabecular meshwork cells. Int J Clin Exp Pathol 7: 4827-4836, 2014

59. Huang X, Li M, Guo X, Li S, Xiao X, Jia X, Liu X and Zhang Q: Mutation analysis of seven known glaucoma-associated genes in Chinese patients with glaucoma. Invest Ophthalmol Vis Sci 55: 3594-3602, 2014.

60. Park J, Kim M, Park CK, Chae H, Lee S, Kim Y, Jang W, Chi HY, Park HY and Park SH: Molecular analysis of myocilin and optineurin genes in Korean primary glaucoma patients. Mol Med Rep 14: 2439-2448, 2016.

61. Maurya N, Agarwal NR and Ghosh I: Low-dose rotenone exposure induces early senescence leading to late apoptotic signaling cascade in human trabecular meshwork (HTM) cell line: An in vitro glaucoma model. Cell Biol Int 40: 107-120, 2016

62. Pattabiraman PP and Rao PV: Hic-5 regulates actin cytoskeletal reorganization and expression of fibrogenic markers and myocilin in trabecular meshwork cells. Invest Ophthalmol Vis Sci 56: 5656-5669, 2015.

63. Wei X, Cho KS, Thee EF, Jager MJ and Chen DF: Neuroimmflammation and microglia in glaucoma: Time for a paradigm shift. J Neurosci Res, 2018. (Epub ahead of print).

64. Wareham LK, Buys ES and Sappington RM: The nitric oxide-guanylate cyclase pathway and glaucoma. Nitric Oxide 77: $75-87,2018$.

65. Michelessi M, Bicket AK and Lindsley K: Cyclodestructive procedures for non-refractory glaucoma. Cochrane Database Syst Rev 4: CD009313, 2018.

66. Stamer WD and Acott TS: Current understanding of conventional outflow dysfunction in glaucoma. Curr Opin Ophthalmol 23 135-143, 2012

67. Kim BS, Savinova OV, Reedy MV, Martin J, Lun Y, Gan L, Smith RS, Tomarev SI, John SW and Johnson RL: Targeted disruption of the myocilin gene (Myoc) suggests that human glaucoma-causing mutations are gain of function. Mol Cell Biol 21: 7707-7713, 2001

68. Acott TS, Kelley MJ, Keller KE, Vranka JA, Abu-Hassan DW, Li X, Aga M and Bradley JM: Intraocular pressure homeostasis: Maintaining balance in a high-pressure environment. J Ocul Pharmacol Ther 30: 94-101, 2014.

69. Fautsch MP, Bahler CK, Jewison DJ and Johnson DH: Recombinant TIGR/MYOC increases outflow resistance in the human anterior segment. Invest Ophthalmol Vis Sci 41: 4163-4168, 2000.

70. Patel GC, Liu Y, Millar JC and Clark AF: Glucocorticoid receptor GR $\beta$ regulates glucocorticoid-induced ocular hypertension in mice. Sci Rep 8: 862, 2018.

71. Faralli JA, Dimeo KD, Trane RM and Peters D: Absence of a secondary glucocorticoid response in C57BL/6J mice treated with topical dexamethasone. PLoS One 13: e0192665, 2018.

72. Nazir S, Mukhtar M, Shahnawaz M, Farooqi S, Fatima N, Mehmood R and Sheikh N: A novel single nucleotide polymorphism in exon 3 of MYOC enhances the risk of glaucoma. PLoS One 13: e0195157, 2018.

73. Shepard AR, Jacobson N, Millar JC, Pang IH, Steely HT, Searby CC, Sheffield VC, Stone EM and Clark AF Glaucoma-causing myocilin mutants require the Peroxisomal targeting signal-1 receptor (PTS1R) to elevate intraocular pressure. Hum Mol Genet 16: 609-617, 2007.

74. Guan Y, Li J, Zhan T, Wang JW, Yu JB and Yang L: Idebenone maintains survival of mutant myocilin cells by inhibiting apoptosis. Chin Med J (Engl) 129: 2001-2004, 2016.

75. Nag A, Lu H, Arno M, Iglesias AI, Bonnemaijer P, Broer L, Uitterlinden AG, Klaver CC, van Duijn C, Hysi PG and Hammond CJ: Evaluation of the myocilin mutation gln368stop demonstrates reduced penetrance for glaucoma in european populations. Ophthalmology 124: 547-553, 2017.

76. Lam DS, Leung YF, Chua JK, Baum L, Fan DS, Choy KW and Pang CP: Truncations in the TIGR gene in individuals with and without primary open-angle glaucoma. Invest Ophthalmol Vis Sci 41: 1386-1391, 2000.
77. Morissette J, Clépet C, Moisan S, Dubois S, Winstall E, Vermeeren D, Nguyen TD, Polansky JR, Côté G, Anctil JL, et al: Homozygotes carrying an autosomal dominant TIGR mutation do not manifest glaucoma. Nat Genet 19: 319-321, 1998

78. Kuchtey J, Chowdhury UR, Uptegraft CC, Fautsch MP and Kuchtey RW: A de novo MYOC mutation detected in juvenile open angle glaucoma causes non-secretion of associated with reduced myocilin protein in aqueous humor. Eur J Med Genet 56 292-296, 2013

79. Huard DJE, Crowley VM, Du Y, Cordova RA, Sun Z, Tomlin MO, Dickey CA, Koren J III, Blair L, Fu H, et al: Trifunctional high-throughput screen identifies promising scaffold to inhibit Grp94 and treat myocilin-associated glaucoma. ACS Chem Biol 13: 933-941, 2018.

80. Stothert AR, Suntharalingam A, Huard DJ, Fontaine SN, Crowley VM, Mishra S, Blagg BS, Lieberman RL and Dickey CA: Exploiting the interaction between Grp94 and aggregated myocilin to treat glaucoma. Hum Mol Genet 23: 6470-6480, 2014

81. Caballero $\mathrm{M}$ and Borras $\mathrm{T}$ : Inefficient processing of an olfactomedindeficient myocilin mutant: Potential physiological relevance to glaucoma. Biochem Biophys Res Commun 282: 662-670, 2001.

82. Vollrath D and Liu Y: Temperature sensitive secretion of mutant myocilins. Exp Eye Res 82: 1030-1036, 2006.

83. Yam GH, Gaplovska-Kysela K, Zuber C and Roth J: Aggregated myocilin induces russell bodies and causes apoptosis: Implications for the pathogenesis of myocilin-caused primary open-angle glaucoma. Am J Pathol 170: 100-109, 2007.

84. Joe MK, Sohn S, Hur W, Moon Y, Choi YR and Kee C: Accumulation of mutant myocilins in ER leads to ER stress and potential cytotoxicity in human trabecular meshwork cells. Biochem Biophys Res Commun 312: 592-600, 2003.

85. Gould DB, Miceli-Libby L, Savinova OV, Torrado M, Tomarev SI, Smith RS and John SW: Genetically increasing Myoc expression supports a necessary pathologic role of abnormal proteins in glaucoma. Mol Cell Biol 24: 9019-9025, 2004.

86. Joe MK, Nakaya N, Abu-Asab M and Tomarev SI: Mutated myocilin and heterozygous Sod 2 deficiency act synergistically in a mouse model of open-angle glaucoma. Hum Mol Genet 24: 3322-3334, 2015

87. Joe MK and Tomarev SI: Expression of myocilin mutants sensitizes cells to oxidative stress-induced apoptosis: Implication for glaucoma pathogenesis. Am J Pathol 176: 2880-2890, 2010.

88. Hill SE, Donegan RK and Lieberman RL: The glaucoma-associated olfactomedin domain of myocilin forms polymorphic fibrils that are constrained by partial unfolding and peptide sequence. J Mol Biol 426: 921-935, 2014.

89. Zode GS, Kuehn MH, Nishimura DY, Searby CC, Mohan K, Grozdanic SD, Bugge K, Anderson MG, Clark AF, Stone EM and Sheffield VC: Reduction of ER stress via a chemical chaperone prevents disease phenotypes in a mouse model of primary open angle glaucoma. J Clin Invest 121: 3542-3553, 2011.

90. Maddineni P, Kasetti RB and Zode GS: Methods for analyzing endoplasmic reticulum stress in the trabecular meshwork of glaucoma models. Methods Mol Biol 1695: 121-134, 2018.

91. Chong WC, Shastri MD and Eri R: Endoplasmic reticulum stress and oxidative stress: A vicious nexus implicated in bowel disease pathophysiology. Int J Mol Sci 18: E771, 2017.

92. Plaisance V, Brajkovic S, Tenenbaum M, Favre D, Ezanno H, Bonnefond A, Bonner C, Gmyr V, Kerr-Conte J, Gauthier BR, et al: Endoplasmic reticulum stress links oxidative stress to impaired pancreatic beta-cell function caused by human oxidized LDL. PLoS One 11: e0163046, 2016.

93. Zhao J, Wang S, Zhong W, Yang B, Sun L and Zheng Y: Oxidative stress in the trabecular meshwork (Review). Int J Mol Med 38: 995-1002, 2016.

94. Grootjans J, Kaser A, Kaufman RJ and Blumberg RS: The unfolded protein response in immunity and inflammation. Nat Rev Immunol 16: 469-484, 2016.

95. Luo K and Cao SS: Endoplasmic reticulum stress in intestinal epithelial cell function and inflammatory bowel disease. Gastroenterol Res Pract 2015: 328791, 2015.

96. Peters JC, Bhattacharya S, Clark AF and Zode GS: Increased endoplasmic reticulum stress in human glaucomatous trabecular meshwork cells and tissues. Invest Ophthalmol Vis Sci 56: 3860-3868, 2015.

97. Huard DJ and Lieberman RL: Progress toward development of a proteostasis drug for myocilin-associated glaucoma. Future Med Chem 10: 1391-1393, 2018. 
98. Mishra SJ, Ghosh S, Stothert AR, Dickey CA and Blagg BS: Transformation of the non-selective aminocyclohexanol-based Hsp90 inhibitor into a Grp94-seletive scaffold. ACS Chem Biol 12: 244-253, 2017.

99. Crowley VM, Khandelwal A, Mishra S, Stothert AR, Huard DJ, Zhao J, Muth A, Duerfeldt AS, Kizziah JL, Lieberman RL, et al: Development of glucose regulated protein 94 -selective inhibitors based on the BnIm and radamide scaffold. J Med Chem 59: 3471-3488, 2016

100. Stothert AR, Suntharalingam A, Tang X, Crowley VM, Mishra SJ, Webster JM, Nordhues BA, Huard DJE, Passaglia CL, Lieberman RL, et al: Isoform-selective Hsp90 inhibition rescues model of hereditary open-angle glaucoma. Sci Rep 7: 17951, 2017.

101. Keller KE and Wirtz MK: Working your SOCS off: The role of ASB10 and protein degradation pathways in glaucoma. Exp Eye Res 158: 154-160, 2017.

102. Wang N, Chintala SK, Fini ME and Schuman JS: Activation of a tissue-specific stress response in the aqueous outflow pathway of the eye defines the glaucoma disease phenotype. Nat Med 7: 304-309, 2001.

103. Yerramothu P, Vijay AK and Willcox MDP: Inflammasomes the eye and anti-inflammasome therapy. Eye (Lond) 32: 491-505, 2018.

104. Meier-Soelch J, Jurida L, Weber A, Newel D, Kim J, Braun T, Schmitz ML and Kracht M: RNAi-based identification of gene-specific nuclear cofactor networks regulating interleukin-1 target genes. Front Immunol 9: 775, 2018.

105. Yasuda M, Takayama K, Kanda T, Taguchi M, Someya $\mathrm{H}$ and Takeuchi M: Comparison of intraocular pressure-lowering effects of ripasudil hydrochloride hydrate for inflammatory and corticosteroid-induced ocular hypertension. PLoS One 12: e0185305, 2017.

106. Itakura T, Peters DM and Fini ME: Glaucomatous MYOC mutations activate the IL-1/NF- $\kappa$ B inflammatory stress response and the glaucoma marker SELE in trabecular meshwork cells. Mol Vis 21: 1071-1084, 2015.

107. Kasetti RB, Phan TN, Millar JC and Zode GS: Expression of mutant myocilin induces abnormal intracellular accumulation of selected extracellular matrix proteins in the trabecular meshwork. Invest Ophthalmol Vis Sci 57: 6058-6069, 2016.

108. Donegan RK, Hill SE, Freeman DM, Nguyen E, Orwig SD, Turnage KC and Lieberman RL: Structural basis for misfolding in myocilin-associated glaucoma. Hum Mol Genet 24: 2111-2124, 2015.

109. Joe MK, Sohn S, Choi YR, Park H and Kee C: Identification of flotillin-1 as a protein interacting with myocilin: Implications for the pathogenesis of primary open-angle glaucoma. Biochem Biophys Res Commun 336: 1201-1206, 2005.

110. Joe MK, Kwon HS, Cojocaru R and Tomarev SI: Myocilin regulates cell proliferation and survival. J Biol Chem 289: 10155-10167, 2014

111. Kessenbrock K, Wang CY and Werb Z: Matrix metalloproteinases in stem cell regulation and cancer. Matrix Biol 46: 184-190, 2015.

112. De Groef L, Van Hove I, Dekeyster E, Stalmans I and Moons L: MMPs in the neuroretina and optic nerve: Modulators of glaucoma pathogenesis and repair? Invest Ophthalmol Vis Sci 55: 1953-1964, 2014.
113. Ashworth Briggs EL, Toh T, Eri R, Hewitt AW and Cook AL: TIMP1 TIMP2 and TIMP4 are increased in aqueous humor from primary open angle glaucoma patients. Mol Vis 21: $1162-1172,2015$

114. Filla MS, Dimeo KD, Tong T and Peters DM: Disruption of fibronectin matrix affects type IV collagen fibrillin and laminin deposition into extracellular matrix of human trabecular meshwork (HTM) cells. Exp Eye Res 165: 7-19, 2017.

115. Zode GS, Sharma AB, Lin X, Searby CC, Bugge K, Kim GH, Clark AF and Sheffield VC: Ocular-specific ER stress reduction rescues glaucoma in murine glucocorticoid-induced glaucoma. J Clin Invest 124: 1956-1965, 2014.

116. Ho H, Htoon HM, Yam GH, Toh LZ, Lwin NC, Chu S, Lee YS, Wong TT and Seet LF: Altered anterior segment biometric parameters in mice deficient in SPARC. Invest Ophthalmol Vis Sci 58: 386-393, 2017.

117. Hill SE, Nguyen E, Donegan RK, Patterson-Orazem AC, Hazel A, Gumbart JC and Lieberman RL: Structure and misfolding of the flexible tripartite coiled-coil domain of glaucoma-associated myocilin. Structure 25: 1697-1707, 2017.

118. Burns JN, Turnage KC, Walker CA and Lieberman RL: The stability of myocilin olfactomedin domain variants provides new insight into glaucoma as a protein misfolding disorder. Biochemistry 50: 5824-5833, 2011.

119. Burns JN, Orwig SD, Harris JL, Watkins JD, Vollrath D and Lieberman RL: Rescue of glaucoma-causing mutant myocilin thermal stability by chemical chaperones. ACS Chem Biol 5: 477-487, 2010.

120. Honda R: Role of the disulfide bond in prion protein amyloid formation: A thermodynamic and kinetic analysis. Biophys J 114 885-892, 2018

121. Fingert JH, Héon E, Liebmann JM, Yamamoto T, Craig JE, Rait J, Kawase K, Hoh ST, Buys YM, Dickinson J, et al: Analysis of myocilin mutations in 1703 glaucoma patients from five different populations. Hum Mol Genet 8: 899-905, 1999.

122. Shimizu S, Lichter PR, Johnson AT, Zhou Z, Higashi M, Gottfredsdottir M, Othman M, Moroi SE, Rozsa FW, Schertzer RM, et al: Age-dependent prevalence of mutations at the GLC1A locus in primary open-angle glaucoma. Am J Ophthalmol 130: 165-177, 2000.

123. Millá E, Mañé B, Duch S, Hernan I, Borràs E, Planas E, Dias Mde S, Carballo M, Gamundi MJ and Spanish Multicenter Glaucoma Group-Estudio Multicéntrico Español de Investigación Genética del Glaucoma, EMEIGG: Survey of familial glaucoma shows a high incidence of cytochrome P450 family 1 subfamily B polypeptide 1 (CYP1B1) mutations in non-consanguineous congenital forms in a Spanish population. Mol Vis 19: 1707-1722, 2013.

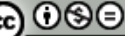

This work is licensed under a Creative Commons Attribution-NonCommercial-NoDerivatives 4.0 International (CC BY-NC-ND 4.0) License. 